\title{
Role of Skills for Clinical, Hospital and Community Pharmacist in Pharmaceutical Care
}

\author{
Anantha Naik Nagappa ${ }^{1 *}$, Vinuth Chikkamath ${ }^{2}$, Vaishnavi Naik ${ }^{2}$, Alisiya M Thomas ${ }^{2}$ and Krithi Krishnan L ${ }^{2}$ \\ ${ }^{1}$ Amity Institute of Pharmacy, Amity University of Madhya Pradesh,India \\ ${ }^{2}$ Department of Pharmacy Practice, SCS College of Pharmacy, India
}

Submission: August 29, 2018; Published: October 15, 2018

*Corresponding author: Anantha Naik Nagappa, Amity Institute of Pharmacy, Amity University of Madhya Pradesh, Gwalior, Madhya Pradesh, India; Email: anantha1232000@gmail.com

Abstract

Due to increased evidence of long term safety issues, the careless use of medication is not only risky but dangerous. It is well established that all the drugs are having potential to cause injuries with long term use. The habits of self-medication are to be replaced by informed selfmedication by qualified pharmacist in the community pharmacy. In hospitals, the contributions of clinical pharmacist and hospital pharmacist, is been found to result into a win-win benefits to all stakeholders like hospitals, individual Doctors, Nurses and Patients. There are in most need to identify important skills and create a conducive atmosphere to develop best pharmacy practice services. In this article, an attempt has been made to highlight the skills for development of delivering pharmaceutical care in clinical, hospital and community settings.

Keywords:Pharmacist; Hospital; Skills; Diagnosing; Nurses; Health care

\section{Introduction}

The pharmacists are intermediate professionals linking physicians, Nurses, and other health care professionals.In the system of health care delivery, the emphasis is on equity for all health care providers to identify their domain of services. The doctors are dedicated to diagnosing and prescribe the medicines with consultation of clinical pharmacist. The Nurses are supposed to provide the nursing to the patients and collaborate with the Doctors and Pharmacist in the therapy.Pharmacists and Nurses are engage in the several activities in monitoring the patients' health condition and documenting the services provided. The pharmacist participates in the health care delivery in three major areas as Clinical services, Hospital services and Community services. In providing such services have to collaborate with the Doctor and Nurse. He has to interact with patients and their attendees to provide health care delivery. The task of health care delivery has to be a team work with integrated communications to achieve excellence in healthcare delivery. In this article, the emergency of pharmaceutical care and its growth in health care delivery is becoming ambiguous across all the services of healthcare delivery[1-3].

i. Skills for Clinical pharmacy services

ii. Skills for Hospital pharmacy services

iii. Skills for Community pharmacy services

\section{Skills for Clinical Pharmacy Services}

Clinical Pharmacist is a core member of the clinical team, who is the responsible for attending the inpatients in Hospital.
In the general ward the patients who are admitted to medicine, Nephrology, cardiology, skin, pediatrics, orthopedics and other patients for treatments are attended by the clinical pharmacist. Patients are also admitted in an intensive care unit (ICU) and special wards are also attended by clinical pharmacist. The prescription review, dose calculations, drug-drug interactions, drug-food interactions, drug allergy and soap analysis are examined by the qualified clinical pharmacist. Clinical pharmacist shall participate in ward rounds and provide the drug information services to doctors and nurses. They are also expected to take the medication history of the patients with newly admitted to the hospital and also provide discharge medication advice. They are responsible to publish and update the hospital formulary and treatment protocols. He is also coordinator for Drugs and Therapeutics Committee; where in recommendations for inclusion of drugs is decided upon based on the merits, availability and price of the drugs. They should also provide the services of Therapeutic drug monitoring services on demand. These services are to be given as soon as possible, and there should be quality in services, the accuracy of information is going to affect the treatments course, cost, and outcomes of treatment [4,5].

The clinical pharmacist is supposed to deliver quickly the correct information with zero errors as the input affects the outcome of therapy. He should practice regularly the search methodology from the internet and databases. It is very important that he must update the knowledge in a systematic fashion. There are free and paid databases of drug information which need to be understood. The effective communication is a basic requirement 


\section{Global Journal of Pharmacy \& Pharmaceutical Sciences}

along with maintaining brevity in the volume of information. The dose calculations are very important as the doctors are busy in diagnosing and nurses in patient care. The skills of calculating the dose depending on the patient specifications like body weight, height, gender, and age play an important role in customizing the number of drugs to be administered. The checking of drug interaction with drugs and food is a routine activity a clinical pharmacist must carry out.

The prescribing information must be put in the drug information system and shall be getting the information needed One should be careful of search term as the response depends on the question/search term fed to the database. Drug information services are another responsibility a clinical pharmacist has to provide. Here he should be able to assess who, why and what time the information is to be delivered. If the information request is from an ICU/Emergency ward, it should be answered in priority. The team of health care provider is waiting for the information based on which they are going to take decisions. If the information is from wards, then the information required may not be very immediate, and shall be about dose, disease or any precautions. It may be the duty doctor, nurse or patients. Now-a-days, handheld devices of drug information are available; with these ones can get the information, the process by screening and provide the information. Understanding the nature of the query and providing the optimized information is the skill, a clinical pharmacist should concentrate upon[6].

Therapeutic drug monitoring is a highly important activity that a clinical pharmacist should provide. There are drugs with very narrow therapeutic index and low margin of safety, for such drugs, it becomes all the more important to maintain the blood concentrations well within the boundaries of therapeutic window. For example: Digitalis, Phenytoin and Antibiotics like Gentamycin and mood stabilizers like Lithium carbonate[7].

\section{Skills for Hospital Pharmacist}

Hospital Pharmacy is having a unique role in managing the issues regarding providing the quality medicines to the therapeutic team on demand. They should be familiar with the marketed products that are being used in the hospitals. The drugs come at varied prices depending on the manufacturer. In fact, the manufacturer may market the product in brand and generic versions. They may also sell the formulations in bulk packs. It may be cheaper for the hospital to buy bulk packs instead of regular packs and save money for the hospital. The hospital pharmacy should implement inventory control of medicine stocks of the hospital. All medicines are perishable and hence smart indenting and establishing an active stock verification is a fundamental requirement. There should be good watch and ward activity on the ordering system. They also should be able to maintain the expiry check, and removal from dispensing section much before the stock crosses the date of expiry[8].

The patients admitted in hospital are in a delicate situation of health. There are some special population, patients like neonates, pediatrics, pregnant women, cancer patients and geriatric. All the medicine required by special population may not be available in the open market. In such conditions with a special license from Drug regulators, one can establish compounding practices in hospital pharmacy. Here the pharmacist prepares the formulation on a prescription and dispenses. He may also prepare extemporous formulations which are to be freshly made for immediate consumption. Here Hospital pharmacist should be able to prepare the formulation on individual prescription. The hospital pharmacy can also make medicines in bulk to be used in the hospitals. For example, Intravenous fluids like normal saline, dextrose normal saline, cough mixture, ointments, and suppository.

\section{Skills for Community Pharmacist}

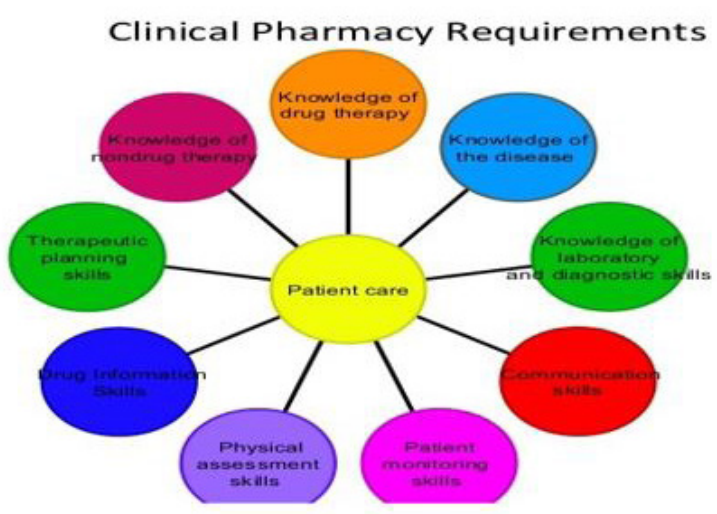

Figure 1: Knowledge and Skill requirements of a Pharmacist.

Community Pharmacy is a place where the patients bring the prescriptions written by the doctors for dispensing the medications. The community pharmacist shall be conducting the act of business. The peer competition among community pharmacy can bring the best practices to attract good number of prescriptions. Community pharmacy may be independent or a chain pharmacy. Here the pharmacist has to scrutinize the prescription for Indications and disease conditions, dose appropriateness, drug-drug/food interactions, adverse drug reactions and medication errors. The community pharmacist 
can maintain the patient profile in the form of Electronic Health Record, update periodically, on demand he may provide the same to doctors. Such services by community pharmacists are going to be appreciated by fellow health care providers and patients and pays back to pharmacy in terms of customers and referrals for the pharmacy. Community pharmacy are also engaged home based services like home medication reviews[9] (Figure 1\&2).

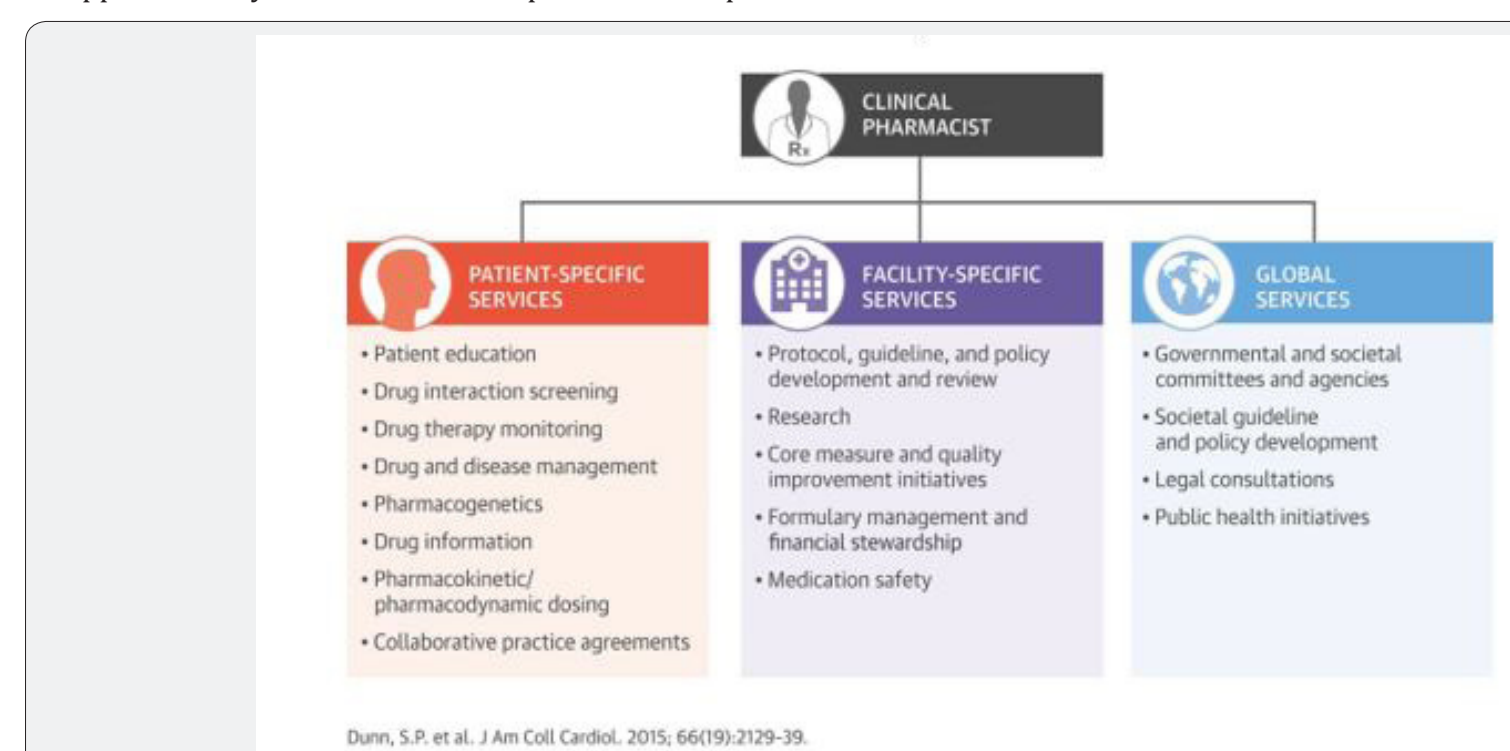

Figure 2: Role of Clinical Pharmacist.

\section{Conclusion}

With the increased complexity of disease, drugs and lifestyle, it has become mandatory to view the safety issues and outcome issues of every treatment. The shift from product centric therapy to patient centric therapy has its origins, when patients were not at all satisfied with clinical outcomes. They demanded the quality of life should be better than before therapy and started asking about the value of therapy in terms of money spent on therapy. The patient requirement can only be served if a pharmaceutical care is provided to all therapies. However, in the past, there were no opportunities for the pharmacist to intervene in the therapy as everything in modern medicine was never used to be questioned. The changed scenario of documentation and accountability has exposed the lacunae in existing health care delivery, which got metamorphosed into patient-centric care. Now through the pharmacy practice one can ensure satisfactory therapy. Hence the skills of pharmacy practice are all that important.

A communication skill helps to precise identifications of the patient's difficulties, the improvement of the level of patient's engagement and approval with the care, and patient's loyalty to treatment. Relationship with patients based on adequate communication decreases patient's anxiety and depression. A patient has his or her own position in the world as a citizen, worker and family member. A disease intimidates such a social background of a patient. One has to realize that patients suffer not only from diseases, but also from psychosocial pain. Such appreciation is the most important basis to improve health communication that creates a worthy relationship with patients.

\section{References}

1. Van Amburgh JA, White RN (2007) A Practical Guide to Pharmaceutical care: A Clinical Skills Primer. Am J Pharm Educ 71(5): 99.

2. Kotegawa T (2007) Communication Skills for Pharmaceutical Care. Yakugaku Zasshi 127(2): 237-244.

3. Monroe DP, Beth S, Edeiken Monroe, Perrier ND (2007) Light and Dark: Surgeons, Radiologists and why they need mutual Understanding to succeed. J Am Coll Surg 205(6): 805-806.

4. Kevin Yi-Lwern Yap, Chan A, Wai Keung Chui (2009) Improving Pharmaceutical Care in Oncology by Pharmaco Informatics: the Evolving Role of Informatics and the Internet for Drug Therapy. Lancet Oncol 10: 1011-1019.

5. McDonough RP (1996) Interventions to Improve Patient Pharmaceutical Care Outcomes. J Am Pharm Assoc (Wash) 36(7): 453-465.

6. Principles of Practice for Pharmaceutical Care (2018).

7. Damian Child, Jonathan Cooke and Richard Hey (2018) Clinical Pharmacy. Sample Chapter Copyright Pharmaceutical Press, London, UK.

8. Charles D Hepler (2004) Clinical Pharmacy, Pharmaceutical Care, and the Quality of Drug Therapy. Pharmacotherapy 24(11): 1491-1498.

9. Rose Marie Parr (2018) Achieving Excellence in Pharmaceutical Care A Strategy for Scotland. NHS Scotland, United Kingdom. 

CC This work is licensed under Creative
Commons Attribution 4.0 License
DOI: $10.19080 /$ GJPPS.2018.06.555684
Your next submission with Juniper Publishers will reach you the below assets

- Quality Editorial service

- Swift Peer Review

- Reprints availability

- E-prints Service

- Manuscript Podcast for convenient understanding

- Global attainment for your research

- Manuscript accessibility in different formats

( Pdf, E-pub, Full Text, Audio)

- Unceasing customer service

Track the below URL for one-step submission https://juniperpublishers.com/online-submission.php 